\title{
Oncofetal Gene SALL4 in Aggressive Hepatocellular Carcinoma
}

\author{
Kol Jia Yong, B.Sc., Chong Gao, M.D., Ph.D., Joline S.J. Lim, M.B., B.S., \\ Benedict Yan, M.B., B.S., Henry Yang, Ph.D., Todor Dimitrov, Ph.D., \\ Akira Kawasaki, M.D., Ph.D., Chee Wee Ong, M.Sc., Kwong-Fai Wong, Ph.D., \\ Sanghoon Lee, Ph.D., Sharada Ravikumar, M.D., Ph.D., Supriya Srivastava, M.D., \\ Xi Tian, B.S., Ronnie T. Poon, M.B., B.S., Ph.D., Sheung Tat Fan, M.D., D.Sc., \\ John M. Luk, D.Med.Sc., Yock Young Dan, M.B., B.S., Ph.D., \\ Manuel Salto-Tellez, M.D., Li Chai, M.D., and Daniel G. Tenen, M.D.
}

From the Cancer Science Institute of Singapore (K.J.Y., H.Y., A.K., C.W.O., K.-F.W., S.L., S.R., S.S., J.M.L., M.S.-T., D.G.T.), the National University of Singapore Graduate School for Integrative Sciences and Engineering (K.J.Y.), the National University Cancer Institute (J.S.J.L.), and the Departments of Pathology (B.Y.), Pharmacology (J.M.L.), and Medicine (Y.Y.D.), National University Health System and National University of Singapore, Singapore; the Department of Pathology, Brigham and Women's Hospital (C.G. J.S.J.L., T.D., X.T., L.C.), and the Harvard Stem Cell Institute (D.G.T.), Harvard Medical School, Boston; the Department of Surgery, Queen Mary Hospital, Hong Kong (R.T.P., S.T.F., J.M.L.); and the Centre for Cancer Research and Cell Biology, Queen's University Belfast, Belfast, United Kingdom (M.S.-T.). Address reprint requests to Dr. Tenen at the Cancer Science Institute of Singapore, 12th Fl., Unit 01, MD6 Center for Translational Medicine, National University of Singapore Yong Loo Lin School of Medicine, 14 Medical Dr., Singapore 117599 , or at daniel .tenen@nus.edu.sg.

Drs. Chai and Tenen contributed equally to this article.

N Engl J Med 2013;368:2266-76. DOI: 10.1056/NEJMoal300297

Copyright @ 2013 Massachusetts Medical Society.

\section{ABSTRACT}

\section{BACKGROUND}

Hepatocellular carcinoma is the third leading cause of cancer-related deaths worldwide. In the heterogeneous group of hepatocellular carcinomas, those with characteristics of embryonic stem-cell and progenitor-cell gene expression are associated with the worst prognosis. The oncofetal gene SALL4, a marker of a subtype of hepatocellular carcinoma with progenitor-like features, is associated with a poor prognosis and is a potential target for treatment.

\section{METHODS}

We screened specimens obtained from patients with primary hepatocellular carcinoma for the expression of SALL4 and carried out a clinicopathological analysis. Loss-of-function studies were then performed to evaluate the role of SALL4 in hepatocarcinogenesis and its potential as a molecular target for therapy. To assess the therapeutic effects of a peptide that targets SALL4, we used in vitro functional and in vivo xenograft assays.

\section{RESULTS}

SALL4 is an oncofetal protein that is expressed in the human fetal liver and silenced in the adult liver, but it is reexpressed in a subgroup of patients who have hepatocellular carcinoma and an unfavorable prognosis. Gene-expression analysis showed the enrichment of progenitor-like gene signatures with overexpression of proliferative and metastatic genes in SALL4-positive hepatocellular carcinomas. Loss-offunction studies confirmed the critical role of SALL4 in cell survival and tumorigenicity. Blocking SALL4-corepressor interactions released suppression of PTEN (the phosphatase and tensin homologue protein) and inhibited tumor formation in xenograft models in vivo.

\section{CONCLUSIONS}

SALL4 is a marker for a progenitor subclass of hepatocellular carcinoma with an aggressive phenotype. The absence of SALL4 expression in the healthy adult liver enhances the potential of SALL4 as a treatment target in hepatocellular carcinoma. (Funded by the Singapore National Medical Research Council and others.) 
EPATOCELLULAR CARCINOMA IS THE third leading cause of cancer-related deaths globally. Although the epidemiologic risk factors for hepatocellular carcinoma are well known, ${ }^{1}$ the molecular mechanisms underlying hepatocarcinogenesis are not well characterized. Elucidation of these mechanisms may allow identification of new candidates for therapeutic targeting. Although surgery, liver transplantation, and radiologic intervention may be viable options for patients with early-stage disease, the prognosis associated with advanced-stage hepatocellular carcinoma remains bleak. ${ }^{2}$ Combination chemotherapy has not improved overall survival but has nonetheless been in wide use for many years because of its possible role in palliation. The need to understand the molecular pathogenesis of hepatocellular carcinoma and develop more effective targeted therapies remains urgent.

The human homologue of the Drosophila spalt homeotic gene, SALL4, encodes a $\mathrm{C} 2 \mathrm{H} 2$ zinc-finger transcription factor. ${ }^{3}$ It is one of the key factors for maintenance of pluripotency and self-renewal of embryonic stem cells. ${ }^{4-6}$ In the murine liver, Sall4 expression diminishes gradually during development and is silenced by adulthood; this suggests a role for Sall4 during early-to-middle development of the fetal liver. ${ }^{7}$ SALL4 is an oncogene that was first described in leukemia. SALL4 was found to be constitutively expressed in human acute myeloid leukemia, and acute myeloid leukemia developed in SALL4B-transgenic mice. ${ }^{8}$ Subsequently, SALL4 expression has been reported in various types of cancers ${ }^{9,10}$ and is proposed to have diagnostic value in several of them.11-13

The progenitor-like subtype of hepatocellular carcinoma is known to be associated with a poor prognosis. ${ }^{14}$ The expression of Sall4 in murine fetal liver but not in adult liver led us to hypothesize that SALL4 might be an important marker for the progenitor-like subtype of hepatocellular carcinoma. We also hypothesized that SALL4 would contribute to the development and persistence of hepatocellular carcinoma when it was expressed in hepatocytes in adults.

\section{METHODS}

\section{PATIENTS AND SAMPLES}

We obtained specimens of hepatocellular carcinoma from patients at the National University Hospital in Singapore (hereafter referred to as the Singapore specimens) to construct hepatocellular-carcinoma tissue microarrays. We also analyzed a second batch of specimens obtained from patients at the Queen Mary Hospital in Hong Kong (hereafter referred to as the Hong Kong specimens). The patients received various treatments and were not participants in any clinical trials evaluating treatment approaches. The institutional review board of the National University of Singapore approved the study, and patients provided written informed consent for the use of all surgically removed tissue specimens used in this study.

\section{STUDY CONDUCT}

The study had no commercial sponsors. The authors vouch for the accuracy and completeness of the data and analysis. No one who is not listed as an author contributed to the manuscript. Four of the authors hold a patent application for intellectual property derived from this study.

\section{STATISTICAL ANALYSIS}

All experiments were performed in triplicate unless otherwise stated. Statistical analyses were performed with the use of SPSS software, versions 15.0 and 16.0 (SPSS). To examine the association between levels of SALL4 expression and clinicopathological characteristics, Fisher's exact test was used for analysis of the Singapore specimens of primary hepatocellular carcinomas and the chi-square test was used for analysis of the Hong Kong specimens. In the Hong Kong specimens, levels of SALL4 expression were divided into two groups. The group with a high level of SALL4 expression (high-SALL4 group) had SALL4 expression intensity that was equal to or exceeded the median level of expression detected on microarray analysis, whereas the group with a low level of SALL4 expression (low-SALL4 group) had SALL4 expression intensity that was lower than the median level of expression. Univariate analyses of cumulative overall survival and tumor recurrence were performed with the use of a Cox proportional-hazards regression model in the Singapore cohort and with the use of the Kaplan-Meier method followed by the log-rank test in the Hong Kong cohort. Factors with prognostic significance were included in the subsequent multivariate analyses with the use of the Cox proportional-hazards regression model in both cohorts. For statistical analysis of the Singapore specimens of primary hepatocellular carcinomas, 
79 of the 179 specimens for which full clinicopathological data were available were analyzed. The correlation significance was determined by means of Spearman and Pearson correlation analyses. The chi-square test and Student's t-test were used for comparisons between the high-SALL4 and low-SALL4 groups. A P value of 0.05 or less was considered to indicate statistical significance.

\section{RESULTS}

SALL4 EXPRESSION IN HEPATOCELLULAR CARCINOMA

To test our hypothesis that SALL4 is reexpressed in a subtype of hepatocellular carcinoma, we examined the expression of SALL4 in liver specimens from patients with and those without hepatocellular carcinomas. With the use of immunohistochemical analysis, we detected SALL4 expression in fetal liver specimens but not in adult liver specimens (Fig. 1A).

We then investigated the level of expression of SALL4 in patients with hepatocellular carcinoma. We constructed a panel of tissue microarrays consisting of 179 surgically resected specimens of primary hepatocellular carcinomas and matched, archived, non-neoplastic liver specimens obtained from the National University Hospital in Singapore. The patients in the Singapore cohort were approximately 56 years of age, $81 \%$ were men, and $87.3 \%$ had grade I or II tumors. Detailed demographic and clinicopathological characteristics of these patients are described in Table S1 in the Supplementary Appendix, available with the full text of this article at NEJM.org. With the use of immunohistochemical analysis, we observed differential expression of SALL4 in matched tissue specimens, with more SALL4expressing cells in the specimens with hepatocellular carcinoma than in the matched non-neoplastic specimens $(\mathrm{P}<0.001)$ (Fig. 1B). Data from the immunohistochemical analysis showed SALL4 positivity in $55.6 \%$ (95 of 171) of the hepatocellular carcinoma specimens analyzed, albeit at various levels of expression (Table S2 in the Supplementary Appendix).

We further confirmed SALL4 up-regulation by means of gene-expression microarray analysis in various independent groups of specimens of primary hepatocellular carcinoma. In a group of 228 matched neoplastic and non-neoplastic liver specimens obtained from patients in Hong Kong,
Figure 1 (facing page). SALL4 Expressed in Human Fetal Liver, Silenced in Adult Liver, and Reactivated in a Subtype of Hepatocellular Carcinoma.

Panel A shows immunohistochemical staining of formalin-fixed, paraffin-embedded human fetal liver and human adult liver. SALL4 expression is indicated by brown staining. Scale bars indicate $100 \mu \mathrm{m}$. Panel B shows immunohistochemical staining of a hepatocellular carcinoma specimen and the matched non-neoplastic liver specimen on tissue microarray analysis. SALL4 expression was detected in the hepatocellular carcinoma tissue and localized in the nucleus, as indicated by the brown staining. Scale bars indicate $100 \mu \mathrm{m}$. Panel C shows differential SALL4 expression in 228 matched specimens of primary hepatocellular carcinoma and non-neoplastic liver tissue from the Hong Kong cohort, as determined by means of microarray analysis. Panel D shows microarray SALL4 expression in primary hepatocellular carcinoma and non-neoplastic liver tissue on the basis of pooled data from public databases. Panel $E$ shows the results of quantitative reverse-transcriptase-polymerase-chain-reaction analysis of SALL4A (left) and SALL4B (right) expression in 10 human hepatocellular-carcinoma cell lines and two immortalized nontransformed hepatocyte cell lines, THLE-2 and THLE-3. All values were normalized to ACTB and plotted relative to the expression of the THLE-2 cell line.

we detected differential SALL4 expression $(\mathrm{P}<0.001)$ (Fig. 1C), which was similar to the pattern of expression observed in the Singapore specimens. Table S3 in the Supplementary Appendix lists the demographic and clinicopathological characteristics of the patients with hepatocellular carcinoma in the Hong Kong cohort. Furthermore, we pooled various global gene-expression data from public databases and observed differential SALL4 expression in the hepatocellular-carcinoma and non-neoplastic liver-tissue specimens $(\mathrm{P}=0.003)$ (Fig. 1D). From these sizable independent groups of adult liver specimens, we confirmed that SALL4 was up-regulated in the subgroup of specimens with hepatocellular carcinoma but remained silenced in the matched non-neoplastic specimens.

Analysis of endogenous SALL4 expression across a panel of 10 human hepatocellular carcinoma cell lines by means of a quantitative polymerase-chain-reaction assay showed the expression of SALL4 at various levels, reflecting the expression pattern observed in the hepatocellular carcinoma tissue specimens described above (Fig. 1E). Moreover, SALL4 expression was not detected in the two immortalized, nontransformed liver-cell lines, THLE-2 and THLE-3. These data 


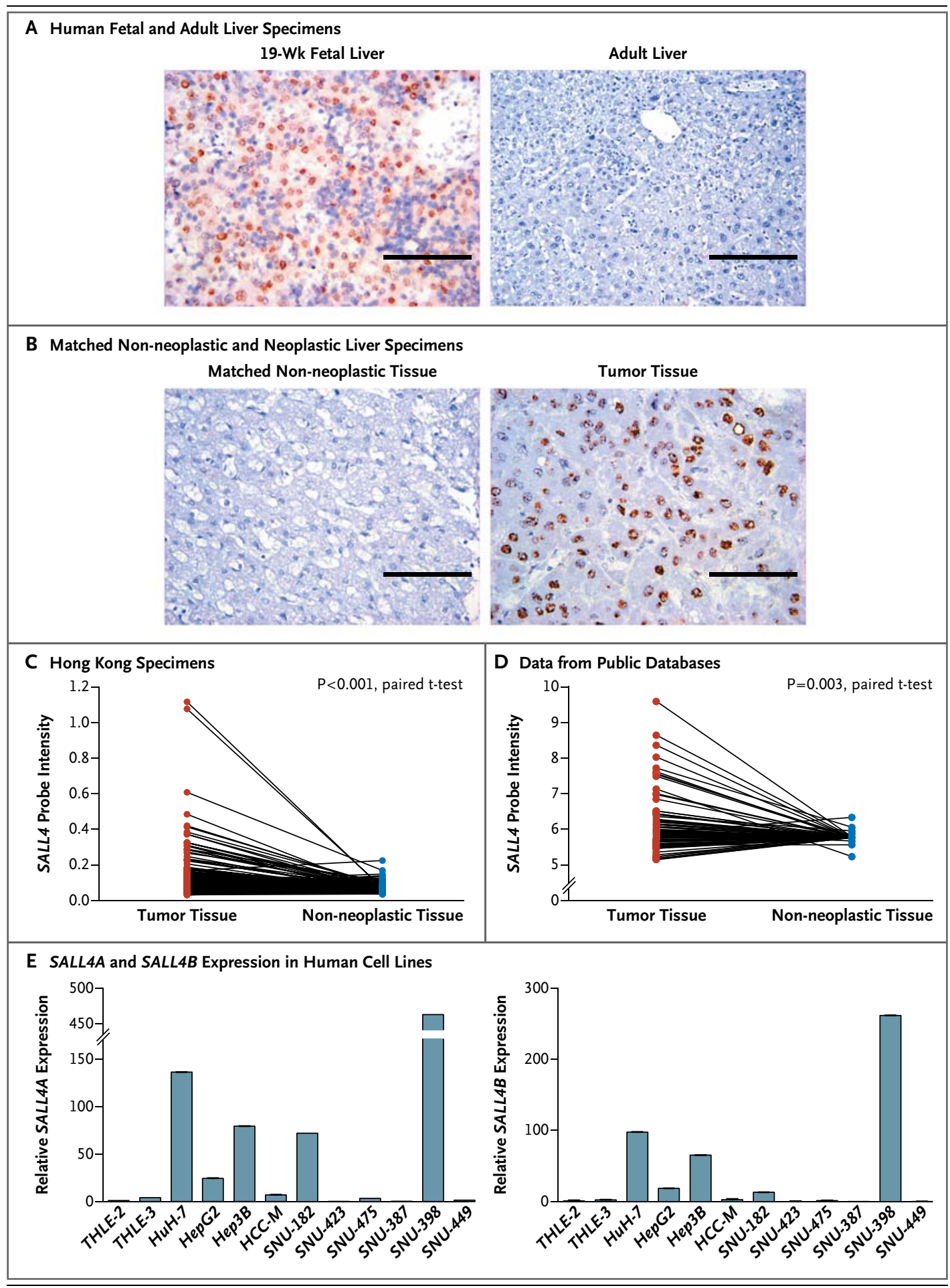

suggest that these cell lines are appropriate models for further testing of our hypothesis and confirm our finding that SALL4 is reexpressed in a subgroup of hepatocellular carcinomas.
In summary, the results obtained from a number of different assays and groups of clinical specimens showed that SALL4 was expressed in fetal liver tissue, silenced in normal 
adult liver tissue, and reexpressed in many of the tissue specimens from adults with hepatocellular carcinoma (Fig. S2 in the Supplementary Appendix).

\section{SALL4 AS A PROGNOSTIC FACTOR} FOR HEPATOCELLULAR CARCINOMA

To analyze the clinical relevance of SALL4 reactivation in hepatocellular carcinoma, we carried out a clinicopathological analysis of the Singapore and Hong Kong specimens of primary hepatocellular carcinoma. This analysis showed that patients with a high level of SALL4 expression had a worse prognosis than patients with a low level of SALL4 expression. A univariate analysis of survival among patients in the Singapore cohort showed that the absence of SALL4 protein (an immunohistochemical score of 0 on a scale of 0 to 4 , with higher scores indicating a greater proportion of positive cells [see the Supplementary Methods section in the Supplementary Appendix]), conferred a significant survival advantage $(\mathrm{P}=0.02)$ (Fig. 2A). Similarly, SALL4 expression was associated with poor survival among the patients in the Hong Kong cohort ( $\mathrm{P}=0.002)$ (Fig. 2B). Our univariate analysis also showed that SALL4 was associated with disease recurrence in both the Singapore cohort (Table S4 in the Supplementary Appendix) and the Hong Kong cohort (Table S5 in the Supplementary Appendix).

In addition, our association studies confirmed that SALL4 expression status was not associated with baseline liver function or the tumor-nodemetastasis and Barcelona Clinic Liver Cancer stages in either the Singapore cohort (Table S1 in the Supplementary Appendix) or the Hong Kong cohort (Table S3 in the Supplementary Appendix). Moreover, SALL4 expression status in the Singapore cohort did not differ significantly according to whether the patients received or did not receive preoperative treatment. In a multivariate Cox regression model, SALL4 was an independent prognostic factor for overall survival (hazard ratio for death, 2.87; 95\% confidence interval [CI], 1.09 to $7.52 ; \mathrm{P}=0.03$ ) in the Singapore cohort (Table S6 in the Supplementary Appendix) and an independent predictor of both overall survival (hazard ratio for death, 1.52; 95\% CI, 1.00 to 2.32; $\mathrm{P}=0.05$ ) and early recurrence (hazard ratio, 1.67; 95\% CI, 1.11 to 2.51; $\mathrm{P}=0.01$ ) in the Hong Kong cohort (Table S7 in the Supplementary Appendix), after adjustment for other clinicopathological features that have conventionally been accepted as having prognostic value in hepatocellular carcinoma.

Gene-profiling studies suggest that patients with cancers that have embryonic stem-cell or hepatic progenitor cell-like gene-expression signatures have a poor prognosis. ${ }^{14-16}$ To investigate whether SALL4-positive hepatocellular carcinomas share a gene-expression pattern with fetal hepatoblasts, we extracted global gene-expression data on hepatocytes, fetal liver tissue, and hepatocellular carcinomas (all from humans) from the Gene Expression Omnibus database for hierarchical cluster analysis. Our analysis showed that high-SALL4 hepatocellular carcinomas clustered tightly with fetal livers, whereas the lowSALL4 hepatocellular carcinomas clustered with normal hepatocytes (Fig. 2C). These findings suggest that hepatocellular carcinomas that express SALL4 have a gene-expression pattern that is similar to the pattern in hepatic progenitor cells and thus are poorly differentiated, aggressive, and associated with a poor prognosis.

We next examined the importance of SALL4 in hepatocellular carcinoma by performing loss-offunction studies. Knocking down SALL4 by means of short hairpin RNA (shRNA) caused a decrease in cell viability, an increase in apoptosis, and a decrease in tumorigenicity of hepatocellular carcinoma cells (Fig. S1 in the Supplementary Appendix). To investigate whether loss of SALL4 in hepatocellular carcinoma cells reverses the aggressive progenitor-like phenotype, we carried out microarray analysis of gene-expression profiles of SNU-398 cells treated with control scrambled shRNA (Scr shRNA 1) or SALL4-specific shRNA (shSALL4 1) and then performed hierarchical clustering of these profiles along with those derived from normal human hepatocytes and fetal liver tissues. As expected, SNU-398 cells treated with scrambled shRNA 1 clustered closely with human fetal liver tissue, since both express high levels of SALL4. SALL4-knocked down SNU-398 cells clustered with human hepatocytes; this suggested that down-regulation of SALL4 can render hepatocellular carcinoma more hepatocyte-like with respect to gene expression (Fig. 2D).

To correlate the subgroup of SALL4-positive 


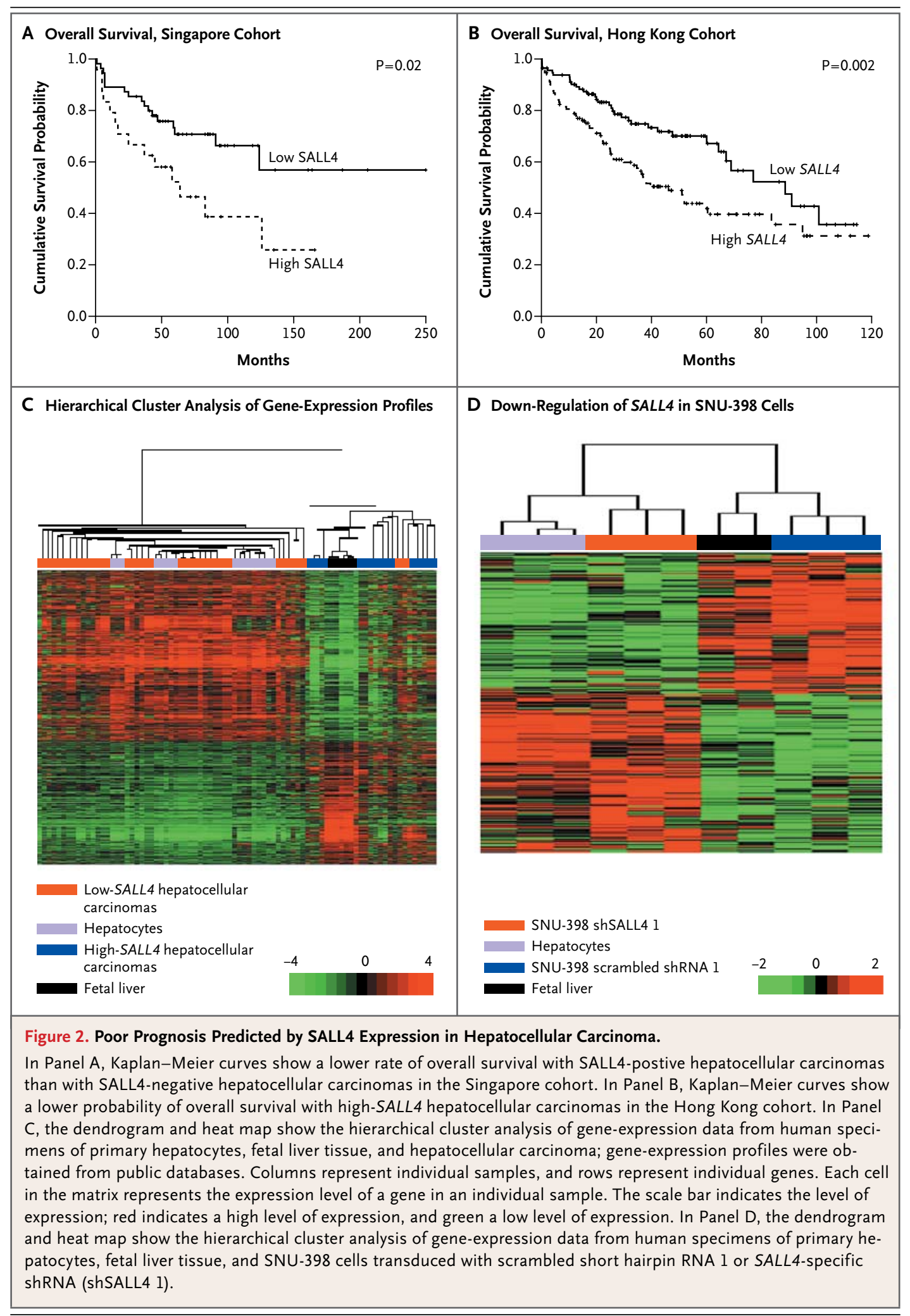

N ENGLJ MED 368;24 NEJM.ORG JUNE 13, 2013

The New England Journal of Medicine

Downloaded from nejm.org on April 22, 2014. For personal use only. No other uses without permission.

Copyright (C) 2013 Massachusetts Medical Society. All rights reserved. 
hepatocellular carcinomas with oncogenic pathways in primary hepatocellular carcinoma specimens, we carried out gene-set enrichment analysis to investigate the enrichment of pathways that have prognostic value in SALL4-high and SALL4low hepatocellular carcinomas. We found that genes that were up-regulated in hepatocellular carcinoma associated with poor survival, genes in an embryonic stem-cell signature, and genes that were up-regulated in metastasis, hepatoblastoma, and a proliferation subclass of hepatocellular carcinoma ${ }^{17}$ were significantly enriched in the high-SALL4 hepatocellular-carcinoma subgroup (Fig. 3A through 3E). Taken together, these data suggest that the expression of oncofetal protein SALL4 can be used as a molecular marker to identify an aggressive progenitor cell-like subtype of hepatocellular carcinoma and that SALL4 expression has prognostic value for patients with hepatocellular carcinoma.

\section{TARGETING SALL4 BY A NOVEL PEPTIDE}

Our loss-of-function studies suggested that SALL4 is a potential therapeutic target for hepatocellular carcinoma and that down-regulation of SALL4 might reverse the aggressive phenotype of hepatocellular carcinomas. We next sought to develop targeted therapy to antagonize the oncogenic role of SALL4. It is known that SALL4 functions as a transcription repressor by recruiting a histone deacetylase (HDAC)-containing nucleosome remodeling and HDAC (NuRD) complex, and that the tumor suppressor PTEN (phosphatase and tensin homologue) is among the target genes repressed by SALL4. ${ }^{19}$ Our SALL4 loss-of-function microarray data provided direct evidence that in hepatocellular carcinoma, the PTEN-AKT pathway is involved in SALL4-induced hepatocarcinogenesis, since the class I phosphatidylinositol 3-kinase (PI3K) signaling events mediated by AKT were significantly affected by SALL4 downregulation $(\mathrm{P}=0.02)$.

We recently described a SALL4 12-AA peptide as a competitive inhibitor that blocks the interaction between SALL4 and NuRD and as a result blocks the NuRD-mediated SALL4-repression function. ${ }^{20}$ We tested whether this 12-AA SALL4 peptide was effective in targeting the SALL4PTEN-AKT pathway and resulted in decreased viability of hepatocellular carcinoma cells. When $5 \mu \mathrm{M}$ or $20 \mu \mathrm{M}$ of nonmutant SALL4 peptide was added to SNU-398 cells, the number of viable
Figure 3 (facing page). Enriched Gene Signatures Associated with a Poor Prognosis in High-SALL4 Hepatocellular Carcinoma.

Enrichment of genes associated with poor survival (Panel A), embryonic stem cells (Panel B), metastasis (Panel C), hepatoblastoma (Panel D), and a proliferation subclass of hepatocellular carcinoma was described by Chiang et al. ${ }^{17}$ (Panel E) in high-SALL4 hepatocellular carcinoma. The heat maps show the enrichment of genes implicated in the gene sets. Columns represent individual samples, and rows represent each gene. Each cell in the matrix represents the expression level of a gene in an individual sample. Red indicates a high level of expression, and green a low level of expression. NES denotes normalized enrichment score in gene-set enrichment analysis. An NES score of more than 1 indicates enrichment of the gene set in high-SALL4 hepatocellular carcinoma. The ranked list metric was generated by calculating the signal-tonoise ratio, ${ }^{18}$ which is based on the difference of means scaled according to the standard deviation. The larger the signal-to-noise ratio, the more distinct the gene expression is in each phenotype and the more the gene acts as a "class marker." The Broad Institute Gene Set Enrichment Analysis website (www.broad.mit.edu/ gsea) provides detailed information about the computational method.

cells was reduced, as compared with SNU-398 cells treated with control mutant peptide and scrambled peptide. Trichostatin A, a general HDAC inhibitor, was used as a positive control drug. ${ }^{21,22}$ When the PTEN inhibitor SF1670 was added to the cells treated with SALL4 peptide, it rescued the phenotype, as evidenced by the maintenance of cell viability (Fig. 4A). These results suggest that PTEN plays an important role in the SALL4 peptide-induced loss of tumor-cell viability in hepatocellular carcinoma. In contrast, SALL4 peptide had no effect on SNU-387 cells with undetectable endogenous SALL4 expression (Fig. 4B). This finding suggests that SALL4 peptide is a specific agent inhibiting SALL4-overexpressing hepatocellular carcinoma cells, with minimal toxic effects on SALL4-negative cells, as compared with the HDAC inhibitor trichostatin A.

To confirm the involvement of the tumor suppressor PTEN in the SALL4 peptide-induced reduction of hepatocellular carcinoma cell viability, we analyzed PTEN expression by means of Western blotting. SALL4 peptide treatment induced an extensive increase in levels of PTEN protein in SNU-398 cells (Fig. 4C). In contrast, SALL4 peptide had a negligible effect on PTEN expression in SNU-387 cells (Fig. 4D). We next exam- 


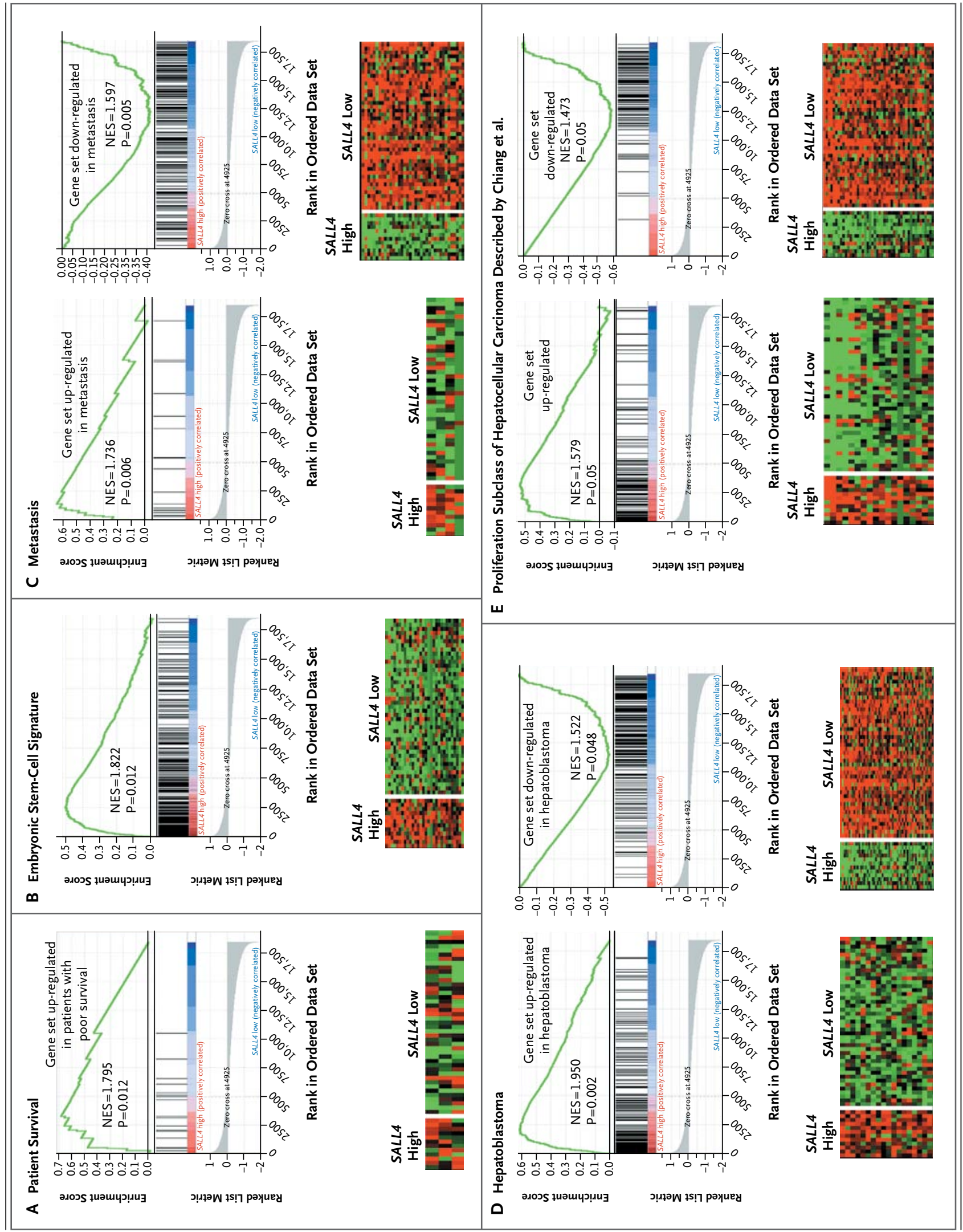




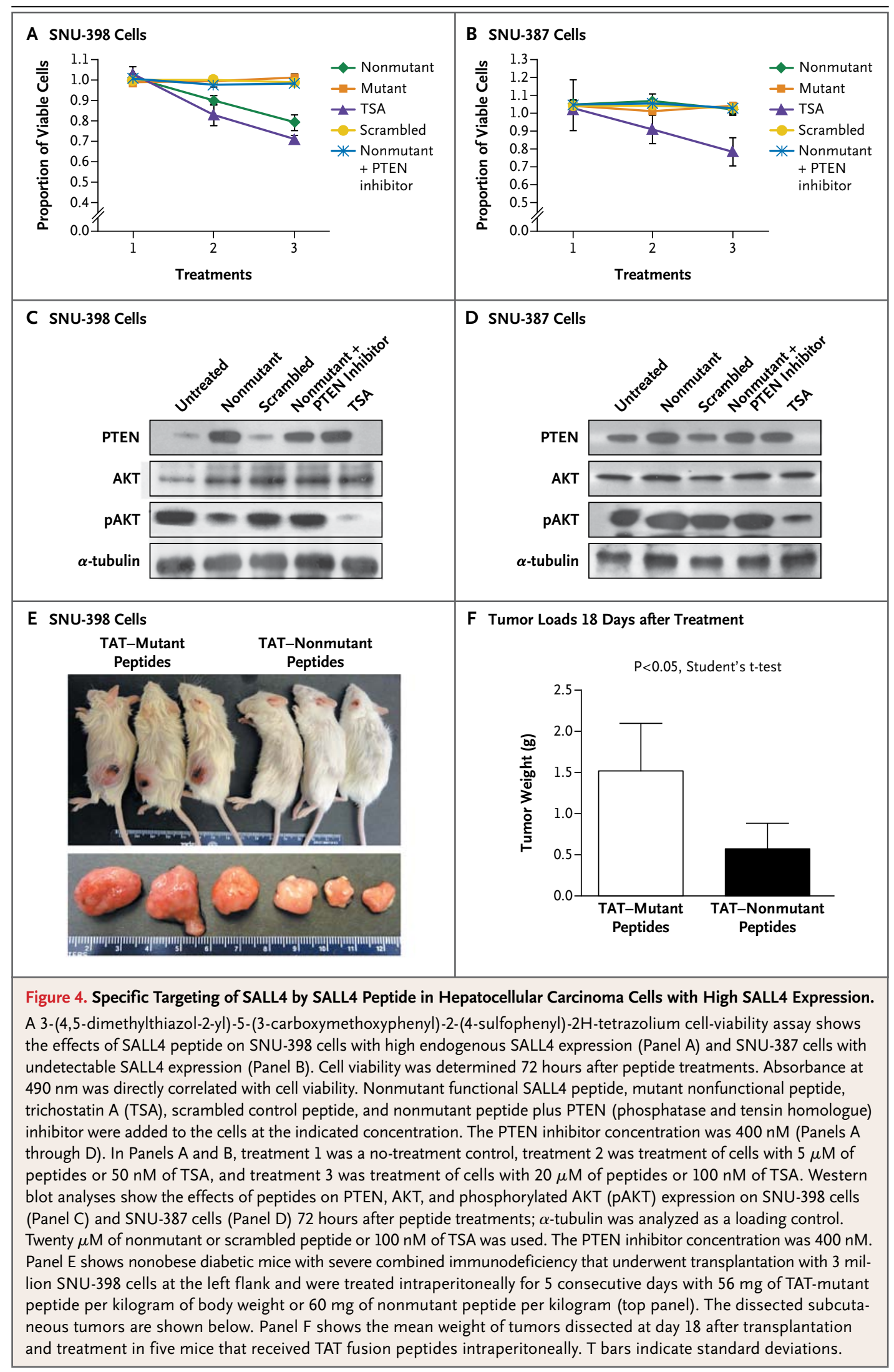


ined whether the increase in PTEN expression had an effect on PI3K signaling. PTEN functions as a phosphatase to dephosphorylate AKT. Western blot analysis showed a marked reduction of phosphorylated AKT protein levels with SALL4 peptide treatment in SNU-398 cells (Fig. 4C), but not in low-SALL4 SNU-387 cells (Fig. 4D); this suggests that the increase in the level of PTEN expression had a functional role in blocking the PI3K survival signaling by dephosphorylating AKT. Furthermore, this effect could be rescued by the PTEN inhibitor, which is consistent with the role of SALL4 in regulating this pathway.

To further test the therapeutic effect of this peptide for in vivo treatment, we conjugated mutant and nonmutant peptides with the transactivator of transcription (TAT) protein transduction domain and administered the peptides intraperitoneally in nonobese diabetic mice with severe combined immunodeficiency, in which SNU-398 cells had been transplanted subcutaneously. TAT fusion nonmutant peptides reduced the tumorigenicity of SNU-398 hepatocellular carcinoma cells (Fig. 4E). The tumor loads at 18 days after treatment in mice that received TAT-nonmutant peptides were significantly smaller than tumor loads in mice that received TAT-mutant peptides (Fig. 4F). These experiments confirm that the SALL4 peptide had biologic activity both in vitro and in vivo in a xenotransplant model.

Our in vitro and murine data suggest that the SALL4 peptide is a potential targeted therapy for a subgroup of hepatocellular carcinomas, since it effectively and specifically reduced viable SALL4positive hepatocellular carcinoma cells.

\section{DISCUSSION}

Hepatocellular carcinoma is one of the deadliest cancers, with a ratio of mortality to incidence of almost $1 .^{1}$ Our study shows the unique expression pattern of the stem-cell factor SALL4 in human livers at various stages - activated in fetal liver tissue, silenced in adult liver tissue, and reexpressed as an oncofetal protein in hepatocellular carcinoma.

Identification of reliable biomarkers that can be used to predict patient outcomes ensures more effective clinical management, and we propose that SALL4 is such a biomarker in hepatocellular carcinoma. The reexpression of SALL4 in hepatocellular carcinoma is clinically significant, since patients with the subtype that overexpresses SALL4 have enriched hepatic progenitor cell-like gene signatures and tend to have a poor prognosis. Given the strong prognostic value of SALL4 established in our retrospective study, we propose that the prognostic value of SALL4 should be further verified in prospective clinical trials.

The discovery of a role for SALL4 in hepatocellular carcinoma, its association with prognosis, and the antitumor effects of a newly identified peptide blocker targeting it have potential therapeutic significance. Testing for the presence of SALL4 at diagnosis may be helpful not only for determining the prognosis but also for identifying patients who are likely to have a response to treatment. Given the expression of SALL4 in hepatocellular carcinoma cells but not in normal adult hepatocytes, treatment with SALL4 peptide may have less tissue toxicity, which is especially beneficial in patients with underlying cirrhosis whose baseline liver function is already compromised. Future studies will determine whether this peptide has synergistic potential with other molecular therapies, such as sorafenib, which act on parallel nonoverlapping pathways.

In conclusion, our study shows that the oncofetal protein SALL4 plays an important role in the extensive network of heterogeneous cellular pathways underlying hepatocarcinogenesis. A 12-amino acid peptide can block the oncogenic role of SALL4 and hence has therapeutic potential in SALL4-positive hepatocellular carcinoma.

Supported by a Singapore Translational Research Award from the Singapore National Medical Research Council (NMRC/ STaR/0001/2008, to Dr. Tenen) and grants from the Singapore Ministry of Education and National Research Foundation (to Dr. Tenen) and the National Institutes of Health (PO1DK080665, to Drs. Tenen and Chai; and RO1HL092437 and PO1HL095489, to Dr. Chai).

Disclosure forms provided by the authors are available with the full text of this article at NEJM.org.

We thank the members of the laboratories of Drs. Tenen and Chai for many helpful discussions and technical support; the Centre for Translational Research and Diagnostics for the generation of microarray data; and Dr. Hongbo R. Luo for discussion about studies of PTEN and AKT.

REFERENCES

1. Ferlay J, Shin HR, Bray F, Forman D, Mathers C, Parkin DM. Estimates of worldwide burden of cancer in 2008: GLOBOCAN 2008. Int J Cancer 2010;127:2893-917.
2. Keating GM, Santoro A. Sorafenib: a review of its use in advanced hepatocellular carcinoma. Drugs 2009;69:22340.
3. de Celis JF, Barrio R. Regulation and function of Spalt proteins during animal development. Int J Dev Biol 2009;53:138598. 
4. Elling U, Klasen C, Eisenberger T, Anlag K, Treier M. Murine inner cell massderived lineages depend on Sall4 function. Proc Natl Acad Sci U S A 2006;103: 16319-24.

5. Yang J, Gao C, Chai L, Ma Y. A novel SALL4/OCT4 transcriptional feedback network for pluripotency of embryonic stem cells. PLoS One 2010;5(5):e10766.

6. Rao S, Zhen S, Roumiantsev S, McDonald LT, Yuan GC, Orkin SH. Differential roles of Sall4 isoforms in embryonic stem cell pluripotency. Mol Cell Biol 2010;30:5364-80.

7. Oikawa $\mathrm{T}$, Kamiya A, Kakinuma S, et al. Sall4 regulates cell fate decision in fetal hepatic stem/progenitor cells. Gastroenterology 2009;136:1000-11.

8. Ma Y, Cui W, Yang J, et al. SALL4, a novel oncogene, is constitutively expressed in human acute myeloid leukemia (AML) and induces AML in transgenic mice. Blood 2006;108:2726-35.

9. Cui W, Kong NR, Ma Y, Amin HM, Lai R, Chai L. Differential expression of the novel oncogene, SALL4, in lymphoma, plasma cell myeloma, and acute lymphoblastic leukemia. Mod Pathol 2006;19:158592.

10. Kobayashi D, Kuribayshi K, Tanaka $M$, Watanabe N. SALL4 is essential for cancer cell proliferation and is overex- pressed at early clinical stages in breast cancer. Int J Oncol 2011;38:933-9.

11. Cao D, Humphrey PA, Allan RW. SALL4 is a novel sensitive and specific marker for metastatic germ cell tumors, with particular utility in detection of metastatic yolk sac tumors. Cancer 2009;115: 2640-51.

12. Wang F, Liu A, Peng Y, et al. Diagnostic utility of SALL4 in extragonadal yolk sac tumors: an immunohistochemical study of 59 cases with comparison to placentallike alkaline phosphatase, alpha-fetoprotein, and glypican-3. Am J Surg Pathol 2009;33:1529-39.

13. Oikawa T, Kamiya A, Zeniya M, et al. Sal-like protein 4 (SALL4), a stem cell biomarker in liver cancers. Hepatology 2013; 57:1469-83.

14. Lee JS, Heo J, Libbrecht L, et al. A novel prognostic subtype of human hepatocellular carcinoma derived from hepatic progenitor cells. Nat Med 2006;12:410-6.

15. Ben-Porath I, Thomson MW, Carey VJ, et al. An embryonic stem cell-like gene expression signature in poorly differentiated aggressive human tumors. Nat Genet 2008;40:499-507.

16. Woo HG, Lee JH, Yoon JH, et al. Identification of a cholangiocarcinoma-like gene expression trait in hepatocellular carcinoma. Cancer Res 2010;70:3034-41.
17. Chiang DY, Villanueva A, Hoshida Y, et al. Focal gains of VEGFA and molecular classification of hepatocellular carcinoma. Cancer Res 2008;68:6779-88.

18. Subramanian A, Tamayo P, Mootha VK, et al. Gene set enrichment analysis: a knowledge-based approach for interpreting genome-wide expression profiles. Proc Natl Acad Sci U S A 2005;102:1554550.

19. Lu J, Jeong H, Kong N, et al. Stem cell factor SALL4 represses the transcriptions of PTEN and SALL1 through an epigenetic repressor complex. PLoS One 2009; 4(5):e5577.

20. Gao C, Dimitrov T, Yong KJ, et al. Targeting transcription factor SALL4 in acute myeloid leukemia by interrupting its interaction with an epigenetic complex. Blood 2013;121:1413-21.

21. Yoshida M, Kijima M, Akita M, Beppu T. Potent and specific inhibition of mammalian histone deacetylase both in vivo and in vitro by trichostatin A. J Biol Chem 1990;265:17174-9.

22. Mukhopadhyay NK, Weisberg E, Gilchrist D, Bueno R, Sugarbaker DJ, Jaklitsch MT. Effectiveness of trichostatin A as a potential candidate for anticancer therapy in non-small-cell lung cancer. Ann Thorac Surg 2006;81:1034-42.

Copyright (๑) 2013 Massachusetts Medical Society.

POSTING PRESENTATIONS FROM MEDICAL MEETINGS ONLINE Online posting of an audio or video recording of an oral presentation at a medical meeting, with selected slides from the presentation, is not considered prior publication. Authors should feel free to call or send e-mail to the Journal's Editorial Offices if there are any questions about this policy. 